\title{
¿ESTRATEGIA REDUCTIVA? DE la ECOlOgía de Sistemas a la Fisiología
}

\author{
FEDERICO DI PASQUO \\ CHRistian FrAnCESE \\ GUILLERMO FOLGUERA
}

\begin{abstract}
The main objective of this work is to analyze the intention to reduce some areas of Ecology (in particular Population Ecology, Community Ecology and Ecosistem Ecology) to Physiology (in the particular case of metabolic studies). The origin of this research of reduction was done through consolidation of metabolic ecological theory in first years of XXIst century. Considering this objective, general framework was based on the proposal of Sahotra Sarkar.
\end{abstract}

Keywords: Reduction; Sarkar; metabolic ecological theory; ecology; physiology.

\section{Introducción}

Sin necesidad de que reciba una presentación, la relación de tipo reductiva ha despertado, en tanto estrategia de unificación, un gran interés entre filósofos de la ciencia y científicos. Más aún, la reducción ha ocupado un lugar importante dentro de la historia de las ciencias (Carnap 1934; Hull 1972; Nagel 2006). Con todo, las estrategias reductivas no han estado exentas de objeciones, a tal punto que muchos autores de distintas corrientes dudan del éxito de estas estrategias (Wimsatt 1976; Darden y Maull 1977; Kitcher 1984; Rosenberg 1985; 1994; Dupré 1993; Burian 1996; Pigliucci y Kaplan 2006; Brigandt y Love 2008; Ferreira y Folguera 2015). En particular, para nuestro estudio descansaremos en la propuesta de reducción sustantiva generada por Sahotra Sarkar (1998; 2001; 2015 y véase también Wimsatt y Sarkar 2006).

En los primeros años del siglo XXI, se ha desarrollado en la ecología un nuevo marco teórico, la teoría metabólica ecológica (TME). En uno de los artículos más relevantes en la temática titulado "Toward a metabolic theory of ecology" (2004), James Brown y colaboradores, ofrecieron precisiones de este conjunto teórico. Sus dos hipótesis centrales son:

(1) Characterizes the effects of body size and temperature on the metabolism of individual organisms ... (Brown et al. 2004a, p.1786).

(2) ... characterizes the effects of metabolism of individual organisms on the pools and flows of energy and matter in populations, communities, and ecosystems. (Brown et al. 2004a, p.1786).

Principia 21(1): 99-123 (2017).

Published by NEL — Epistemology and Logic Research Group, Federal University of Santa Catarina (UFSC), Brazil. 
Con relación a estas hipótesis, la TME presentó una combinación de los efectos de la masa corporal, la temperatura y la estequiometría en relación a la tasa metabólica, mostrando cómo el metabolismo de los individuos puede afectar la estructura y la dinámica de la ecología de sistemas (Brown et al. 2004a; Marquet et al. 2004; Koehl y Wolcott 2004). Es interesante destacar para los fines de este trabajo, que en el marco teórico dado por la TME pueden reconocerse elementos propios de la fisiología directamente exportados hacia el ámbito de la ecología (por ejemplo, el concepto de metabolismo y su relación con la masa corporal y con la temperatura del organismo). Acorde con esto último, debemos destacar también cierta pretensión explicativa que va dirigida desde el dominio de la fisiología hacia los distintos dominios de la ecología (Véase Nuñez et al. 2007; di Pasquo y Folguera 2009; Folguera y di Pasquo 2011). En este trabajo sugerimos entonces, que el metabolismo descripto como una red compleja de reacciones bioquímicas catalizadas por enzimas es considerado un atributo central del organismo. A su vez, destacamos que la tasa metabólica parece operar como una variable reductora desde la cual pueden buscarse las causas de fenómenos registrados en los niveles supra-individuales. Para profundizar el análisis de esta pretendida reducción, a continuación destacamos la justificación que se ha ofrecido para pensar la relación entre el dominio de la fisiología y los dominios de la ecología. En la tercera sección introduciremos la propuesta de Sarkar dirigida a la reducción sustantiva y en las siguientes tres secciones examinaremos los argumentos en favor de esta relación reductiva. Finalmente, ofreceremos algunas consideraciones finales sobre el tipo de reducción efectivamente alcanzada.

\section{El esquema común de lo vivo: una condición necesaria para pensar la relación de reducción entre fisiología y ecología}

Antes de precisar la relación de reducción entre el dominio fisiológico y los dominios señalados de la ecología, resulta interesante introducir un argumento (frecuentemente utilizado por los investigadores que proponen la TME) (Brown et al. 2004a; Brown et al. 2004b; Cottingham y Zens 2004; Marquet et al. 2004) que hace posible pensar en tal relación. En este sentido, se ha discutido si la estimación de variables infraindividuales (propias del dominio fisiológico) pueden ser extrapoladas (o no) de un modo directo al nivel especifico. En caso afirmativo, los resultados obtenidos para un organismo (o un conjunto de estos) se trasladan hacia la especie a la cual pertenece. Para la estrategia referida a dicha extrapolación, se pueden reconocer ciertas consideraciones argumentativas, tal que justifiquen pasar del nivel individual y los niveles infra-individuales (recordemos que el metabolismo del organismo fue caracterizado por un número de reacciones bioquímicas) a niveles supra-individuales. El argumento central descansa en considerar al individuo como representante de la especie bajo 
el supuesto de que el mundo viviente posee un esquema común en cuanto su funcionamiento energético (Spicer y Gaston 1999). Este esquema común en cuanto al funcionamiento energético que subyace a (prácticamente) todas las entidades vivas, puede verse explicitado en distintos trabajos: "In contrast, this chapter builds on the premise that the mechanisms that are responsible for the organization of metabolism are not species-specific" (Kooijman 2000). O bien: "Metabolism sustains life and controls the growth, reproduction, and longevity of living entities" (Marquet et al. 2004, p.1794).

Ahora bien ¿cuáles son las evidencias empíricas presentadas en favor de este esquema fisiológico común según los autores de la TME? En términos generales, se proponen evidencias de que el metabolismo de casi cualquier organismo puede ser explicado y caracterizado a través de la temperatura y de la masa corporal. En este sentido, el rango de la variación metabólica explicada involucra desde los organismos más pequeños hasta lo más grandes (en cuanto a la masa corporal) y desde los más fríos hasta los más calientes (en cuanto a la temperatura):

The explanatory power [...] is substantial, with body size predicting; 100000-fold variation in rates over the 20 orders-of-magnitude size range from the smallest unicellular microbes to the largest vertebrates and trees, and with temperature predicting; $\sim 30$-fold variation over the biologically relevant temperature range from $0^{\circ}$ to $40^{\circ} \mathrm{C}$. (Brown et al. 2004a, p.1775)

Siguiendo esta línea argumental, la "diversidad de lo vivo" quedaría representada por medio de estas dos relaciones (o reglas): la primera, entre el metabolismo y la masa corporal y la segunda, entre el metabolismo y la temperatura corporal. A partir de dicho esquema fisiológico común (representado por estas dos reglas), será posible pasar de la "diversidad fisiológica" a los niveles supra-individuales (los cuales involucran en general, a más de un organismo y distintas especies). Con todo, este esquema común en cuanto a su funcionamiento energético ha generado no pocas controversias entre los ecólogos. Estas controversias, no se han expresado usualmente en los términos de si es legítima (o no) una reducción de la ecología a la fisiología sino más bien, han tomado la forma de una discusión de orden metodológica. Es decir, se han presentado evidencias en contra de este idea de que la "diversidad de lo vivo" posea efectivamente un esquema energético común. Y a su vez, se ha argumentado que este funcionamiento universal es una simplificación excesiva de los sistemas ecológicos (Algar et al. 2007; Hawkins et al. 2007; Muller-Landau et al. 2006).

Habiendo reconocido este esquema común de lo vivo, que permite pensar la relación de reducción entre la fisiología y la ecología y, habiendo precisado las hipótesis de la TME, nos queda la tarea de introducirnos en la relación de reducción antes sugerida. Para ello, a continuación, destacamos la propuesta de reducción sustantiva, desarrollada por Sarkar.

Principia 21(1): 99-123 (2017). 


\section{Elementos para reconocer la reducción "sustantiva" de Sarkar}

En varios de sus trabajos, Sarkar $(1998 ; 2001 ; 2015)$ propone dos tipos de reducción: la formal y la sustantiva. En cuanto a la primera, la cual involucra considerar los aspectos formales de una reducción, el autor se remonta a las tradiciones en que se inscriben las propuestas de Nagel y Woodger. En cuanto a la segunda, la propuesta de reducción sustantiva, descansa en la interpretación de los argumentos científicos, especialmente indagando en lo que asumen acerca del mundo (Sarkar 1998). De aquí que Sarkar agrupe en dos interrogantes los aspectos sustantivos de una reducción:

(i) how is the system that is being studied [and the behavior of which is potentially being explained (or reduced)] represented?; and

(ii) what, exactly, has to be assumed about objects and their interactions for the explanation to work? (Sarkar 1998, p.39).

Así, por medio de estas dos preguntas el autor se distancia de los asuntos formales, a la vez que destaca los aspectos sustantivos que considera de mayor interés. Estos son: el modo en que es representado el sistema y su comportamiento, y qué es lo que se asume acerca de sus objetos y sus interacciones para que una explicación, referida al comportamiento del sistema, funcione. Continuando con las precisiones acerca de la reducción sustantiva y, para llevar adelante la clarificación sobre la relación de reducción entre el dominio de la fisiología y los dominios de la ecología, describiremos los tres criterios de reducción propuestos por Sarkar. En relación con lo dicho, cabe explicitarlos:

(a) Fundamentalismo: Es cuando se reconocen factores y reglas utilizadas para explicar ${ }^{1}$, propias de un dominio considerado más fundamental respecto de otro (sección 4, apartado 1). Y donde las propiedades abordadas puede ser derivadas de estos factores y reglas usando alguna justificación lógica o matemática (4.2) (adaptado de Sarkar 1998, p.43 y 46).

(b) Jerarquía abstracta: Es una jerarquía construida con un criterio independiente, que se asume o bien, se lo tiene por legítimo (sección 5 , apartado 1). Y donde el sentido de la explicación va de los niveles inferiores a los niveles superiores de la jerarquía (5.2) (adaptado de Sarkar 1998, p.43).

(c) Jerarquía espacial: Es cuando la jerarquía abstracta cumple con el criterio físico de "ser una parte espacial de". Esto es, cuando una entidad del nivel inferior es una parte espacial menor de una entidad de nivel superior (sección 6.1) (adaptado de Sarkar 1998, p.43).

Con estas precisiones pasemos entonces a rastrear un conjunto de argumentos que nos permitan validar (o no) estos tres criterios ( $\mathrm{a}, \mathrm{b}$ y c) en el caso de la reducción de la ecología a la fisiología.

Principia 21(1): 99-123 (2017). 


\section{Argumentos a favor de la reducción sustantiva de Sarkar: fundamentalismo}

En esta sección indicaremos algunas evidencias en favor del criterio de fundamentalismo. Para ello, la sección fue dividida en dos apartados. En el primero, reconoceremos factores y reglas explicativas inherentes al dominio considerado fundamental: el dominio fisiológico (sección 4, apartado 1). En el segundo apartado, indicaremos cómo ciertas propiedades inherentes a los dominios de la ecología (de poblaciones, de comunidades y de ecosistemas) pueden ser abordadas, por aquellos factores y reglas mediante alguna justificación matemática o argumental, desde la perspectiva de la TME (4.2).

\subsection{Factores y reglas inherentes al dominio de la fisiología}

En el contexto de la TME se pueden reconocer tres factores cuyo tratamiento será central, para comprender las aproximaciones dirigidas a los dominios de la ecología. El primero de estos factores es el metabolismo, el cual presenta el rol de . ${ }^{\text {articulador. }}{ }^{\text {en }}$ tre el dominio fisiológico y los dominios de la ecología. El metabolismo fue caracterizado como una red compleja de reacciones bioquímicas catalizadas por enzimas: “...the core of metabolism consists of a small number of reactions that form the basis of the TCA (tricarboxylicacid) cycle. The vast majority of organisms use the same basic biochemistry ..." (Brown et al. 2004a, p.1772). Aquí, el metabolismo no sólo es concebido como un conjunto especifico y reducido de reacciones, sino que además, ese conjunto de reacciones resulta inherente a todos los organismos vivos (tal como fue sugerido en la segunda sección). Los otros dos factores son la masa (o el tamaño del cuerpo $)^{2}$ y la temperatura corporal. La TME establece entre estos tres factores (masa, temperatura y metabolismo) ciertas relaciones, que en los términos de Sarkar, van a ser descriptas como reglas. En cuanto a la masa corporal y su relación con el metabolismo (primera regla), Brown y colaboradores (2004a) mencionaban:

Huxley (1932) is credited with pointing out that most size-related variation can be described by so-called alometric equations, which are power functions of the form $\left[Y=Y_{0} M^{b}\right]$ [ec. 1]. They relate some dependent variable, $Y$, such as metabolic rate ${ }^{3}$, development time, population growth rate, or rate of molecular evolution, to body $\mathrm{mass}^{4}, M$, through two coefficients, a normalization constant, $Y_{0}$, and an alometric exponent, $b$. (p.1773).

Así, según los autores de la TME, la tasa metabólica puede ser relacionada con la masa corporal bajo la regla expresada por la ecuación número uno, por lo que buena parte de la variación de la tasa metabólica puede ser explicada por la masa corporal del organismo (ver Figura 1).

Principia 21(1): 99-123 (2017). 
Hasta aquí, hemos precisado cómo es que se relacionan dos de los tres factores considerados (el metabolismo y la masa corporal). Pero ¿qué podemos señalar respecto del otro factor, la temperatura corporal? Es decir, ¿qué relación se presentó entre el metabolismo y la temperatura (segunda regla)? Para dar respuesta a dicha pregunta se recurrió a la ecuación de Boltzmann:

It has been known for more than a century that biochemical reaction rates, metabolic rates, and nearly all other rates of biological activity increase exponentially with temperature. These kinetics are described by the Boltzmann factor or the Van't Hoff-Arrhenius relation $\left[e^{-E / k T}\right]$ [ec.2], where $E$ is the activation energy, $k$ is Boltzmann's constant, and $T$ is absolute temperature in K. (Brown et al. 2004a, p.1773)

Al igual que en el caso de la masa corporal, se puede precisar una relación específica (expresada ahora por la segunda ecuación) entre la temperatura del cuerpo y la tasa metabólica. Tal relación (o regla) representada en la Figura 2, nuevamente viene a dar cuenta de cómo varía la tasa metabólica, pero ahora en función de la temperatura.

Alcanzado este punto conviene volver sobre nuestras propias palabras. Hemos reconocido tres factores (el metabolismo del organismo, la masa y la temperatura corporal) y dos reglas (dadas por las ecuaciones [1] y [2]). A la vez, vale la pena recordar que estos tres factores y estas dos reglas analizadas en el contexto de la TME, resultan inherentes al dominio teórico de la fisiología. A partir de aquí, los autores de la TME pasan a integrar dichas reglas en una única ecuación:

The joint effects of body size, $M$, and temperature, $T$ (in $K$ ), on individual metabolic rate, $I$, can be described by combining [ec. 1 and 2]. This gives $\left[I=i_{0} M^{3 / 4} e^{-E / k T}\right]$ [ec. 3] where $i_{0}$ is a normalization constant independent of body size and temperature. (Brown et al. 2004a, p.1774)

En líneas generales los autores de la TME sugieren que de la integración entre la ecuación que relaciona al metabolismo y la masa corporal $\left[Y=Y_{0} M^{b}\right]$ [ec. 1] con la ecuación que relaciona al metabolismo y la temperatura $\left[e^{-E / k T}\right][$ ec. 2] se obtiene una tercera ecuación $\left[I=I_{0} M^{3 / 4} e^{-E / k T}\right][$ ec. 3], en la cual el metabolismo quedaría explicado mediante las dos reglas antes explicitadas. La obtención de la tercera ecuación resulta central a los fines de nuestro trabajo por diferentes cuestiones. La primera y más trivial, es que vuelve operativa la estimación de la tasa metabólica en el organismo y, la segunda y más importante, es que viene a establecer las reglas a partir de las cuales se podrá pasar del dominio fisiológico a la estimación de otras variables que son inherentes a los dominios de la ecología. De aquí que los abordajes dirigidos al dominio poblacional, comunitario o ecosistémico serán realizados bajo las mismas condiciones en que se han dado los estudios sobre el metabolismo de los organismos. 
Finalmente, cabe resaltar en qué medida los autores de la TME establecen que los tres factores y las dos reglas mencionadas pertenecen a un dominio considerado más fundamental, el fisiológico, respecto de los propios dominios de la ecología. En relación con ello puede leerse que: "Metabolism provides a basis for using first principles of physics, chemistry, and biology to link the biology of individual organisms to the ecology of populations, communities, and ecosystems." (Brown et al. 2004a, p.1771). O en otro ejemplo:

[The Metabolic theory] is based on first principles of energy, mass, and stoichiometric balances [... ] It uses the biological processing of energy and materials to make linkages between individual organisms and the ecology of populations, communities, and ecosystems. (Brown et al. 2004a, p.1786).

De aquí que el metabolismo del organismo - indagado en el dominio de la fisiología -, es el que provee la bases y los principios para "acceder" a los dominios de la ecología. Es en este sentido que el dominio fisiológico se vuelve fundamental respecto de los propios dominios de la ecología.

Hasta aquí hemos visto que: (i) se reconoce un dominio fundamental (el fisiológico) y un conjunto de dominios no fundamentales (el poblacional, el comunitario y el ecosistémico), (ii) se establecen tres factores (el metabolismo, la masa corporal y la temperatura corporal) y (iii) dos reglas (donde se relaciona por un lado, la tasa metabólica del organismo con la masa corporal y por otro, la tasa metabólica con la temperatura corporal) a su vez, estos factores y reglas son recuperados en la ecuación tres. A continuación revisaremos el modo en que son abordados los dominios de la ecología por medio de esta ecuación metabólica.

\subsection{Las propiedades inherentes a los dominios ecológicos son abordadas a partir de la ecuación metabólica [ec. 3]}

En este apartado se busca comprender por medio de los factores y reglas recién presentados, cómo se intentan abordar algunas propiedades inherentes a los dominios ecológicos. Estas propiedades pertenecen específicamente al dominio de la ecología de poblaciones (donde se trabajará sobre las curvas de crecimiento poblacional), al dominio de la ecología de comunidades (donde se abordarán patrones de diversidad de especie) y al dominio de la ecología de ecosistemas (donde se considerarán las ecuaciones de biomasa). Comencemos entonces por el ámbito poblacional.

Dominio poblacional - Una de los modelos más destacados y canónicos de la ecología de poblaciones se dirige a la descripción de la tasa de crecimiento poblacional (Begon et al. 2006; Rockwood 2006). Dicho modelo es presentado comúnmente mediante la siguiente ecuación: $[d N / d t=r . N .(1-N / K)]$ [ec. 4]. Odum y Barrett (2006) describen este modelo poblacional de la siguiente manera: 
$\ldots d N / d t$ es la tasa de crecimiento poblacional (cambio en el número de individuos por unidad de tiempo); $r$ es la tasa intrínseca de crecimiento (el número de nacimientos menos el número de muertes); $N$ es el tamaño de la población (número de individuos) y $K$ es el tamaño máximo posible de la población o capacidad de carga (que viene dado por recursos limitantes por ejemplo: alimento o espacio) (pp.242-3).

Este modelo poblacional predice un crecimiento exponencial hasta que se alcanza un punto de inflexión a partir del cual el crecimiento desacelera. A su vez, otro elemento importante en esta descripción es que el número de individuos presentes en el equilibrio o en la capacidad de carga (cuando $N$ es igual a $K$ ) da por resultado una tasa de crecimiento poblacional igual a cero $[d N / d t=0$ ] [ec. 5]. Según los partidarios de la TME el modelo de crecimiento poblacional en el estado donde el número de individuos se mantiene constante (esto es, cuando $N$ es igual a $K$ ), puede ser explicado mediante los factores antes sugeridos: la masa corporal y la temperatura del organismo y, siguiendo las mismas reglas que fueron utilizadas para la caracterización del metabolismo. En este sentido Brown y colaboradores (2004a) destacaban que:

It is straightforward to solve the equation for population growth rate for the steady state when the number of individuals, $N$, is not changing $(d N / d t=0)$ The equilibrium number of individuals or carrying capacity, $K$, is predicted to vary as $\left[K \sim[R] M^{-3 / 4} e^{E / k T}\right]$ linearly with the $[\ldots]$ concentration of the limiting resource $[R]$, as a power function of body mass, and exponentially with temperature. The qualitative effects of resource supply and body size are not surprising: more individuals with increased resource or decreased size. The effect of temperature, however, may not be so intuitive. Increasing the temperature actually reduces the carrying capacity, because the same supply of energy supports a smaller number of individuals, each fluxing energy and materials at a higher rate. (Brown et al. 2004a, p.1780).

Siguiendo esta larga cita, la tasa de crecimiento poblacional en el estado estacionario puede ser aproximada desde la TME. Así, más individuos son esperados cuando se incrementa el recurso disponible $[R]$ o cuando éstos son más pequeños. En cuanto al efecto de la temperatura, se estiman menos individuos si aumenta la temperatura corporal, dado que estos consumen a mayor velocidad (por que tienen metabolismos más altos) y por tanto, disminuyen más rápidamente los recursos. De aquí que según los autores, se pueda dar cuenta de una de las ecuaciones centrales de la ecología poblacional, la ecuación dirigida al estado estacionario [ec. 5]:

$$
\text { [ec. 5] }[d N / d t=0] \triangleright\left[K \sim[R] M^{-3 / 4} e^{E / k T}\right][\text { ec. 6] }
$$

Recapitulando, se genera a partir de la ecuación de la tasa metabólica [ec. 3] la ecuación número seis, la cual viene a reemplazar a la ecuación de la tasa poblacional en el estado estacionario [ec. 5]. 
Dominio comunitario - En el ámbito de la ecología de comunidades la diversidad de especies es uno de los atributos o propiedades de la comunidad. Justamente, se ha intentado dar cuenta de este atributo recurriendo a la ecuación de la tasa metabólica [ec. 3], la cual considera a la masa y a la temperatura corporal como los factores explicativos. De aquí que los autores se interesen en precisar cómo se relaciona la diversidad de especies con la masa corporal por un lado y por otro, cómo se relaciona la diversidad con la temperatura corporal. En cuanto a la primera de estas relaciones los partidarios de la TME mencionaban:

The fact that species diversity varies inversely with body size suggests that metabolism plays a central role. As recently as a decade ago, the available evidence suggested that the highest diversity occurred in small, but not the smallest, organisms. Recent data, however, reveal enormous microbial diversity and suggest that species richness may continue to increase with decreasing body size right on down to the smallest prokaryotes and perhaps even to viruses. (Brown et al. 2004a, p.1781)

En cuanto a la relación entre la diversidad y la masa corporal los partidarios de la TME, sugieren una relación inversa, esto es, a menor tamaño corporal mayor diversidad de especies. En cuanto a la segunda de estas relaciones mencionadas, dada entre la temperatura y la diversidad, se sostiene que:

It has long been known that diversity of most taxonomic and functional groups is highest in the tropics, but this has usually been attributed to higher productivity $[\ldots]$ or reduced seasonality [...] We have recently shown, however, that species richness in many groups of plants and animals has the same Boltzmann relationship [...] that metabolic rate does. This result holds true not only along latitudinal gradients, but also along elevational gradients $[\ldots]$ The implication is that much of the variation in species diversity is directly attributable to the kinetics of biochemical reactions and ecological interactions. (Brown et al. 2004a, p.1781)

Aquí los autores sugieren que la diversidad de especies y la temperatura se ajustan a la propuesta dada por Boltzmann, la cual recordemos, destaca que un incremento de la temperatura corporal viene acompañado por un aumento en el metabolismo. Una relación similar es la que se propone entre la temperatura y la diversidad de especies, de aquí que un incremento de la temperatura derivaría según estos autores, en un aumento de la diversidad.

Por medio de estas argumentaciones referidas a las relaciones entre la masa y la temperatura corporal con la diversidad de especie, se propone un modelo empírico. ${ }^{5}$ Este modelo, es construido asumiendo las mismas reglas que fueron utilizadas en la caracterización dada para la tasa metabólica:

We hypothesize that diversity is largely a consequence of evolutionary processes that obey Eqs. $\left[B \sim M^{-1 / 4} e^{-E / k T}\right]$ : small or warm organisms having 
faster ecological dynamics than large or cold ones should also have faster evolutionary dynamics, resulting in higher rates of speciation and a higher standing stock of species. (Brown et al. 2004a, pp.1781-2)

De este modo, los patrones de diversidad comunitaria quedan explicados en términos metabólicos por medio de los factores y reglas ya mencionados. Así, mediante pequeñas modificaciones sobre la ecuación número tres se da lugar a la siguiente ecuación: $\left[B \sim M^{-1 / 4} e^{-E / k T}\right.$ ] [ec. 7]. Aquí es importante destacar que en la ecuación siete, el término " $B$ " no es la diversidad de especies sino la tasa de evolución molecular. Dicha aclaración resulta relevante, dado que la diversidad de especies se estima aquí de un modo indirecto, a través de estimaciones dirigidas a las tasas de evolución. Así, la diversidad no presenta una ecuación propia, sino que sería una consecuencia de la tasa de especiación que sí podría ser descripta y explicada por la ecuación siete. De esta manera, se espera que dinámicas evolutivas rápidas - favorecida por organismos pequeños y calientes - deriven en un incremento de la diversidad de especies.

En síntesis, se genera a partir de la ecuación de la tasa metabólica [ec. 3] la ecuación número siete, la cual daría cuenta de las tasas de especiación a partir de donde se podrían derivar los patrones de diversidad.

Dominio ecosistémico - Un último caso puede ser considerado, pero ahora vinculado al dominio de la ecología de ecosistemas. Aquí también sería posible, siguiendo la propuesta de la TME, incorporar modelos empíricos recurriendo a los factores y reglas inherentes al dominio fisiológico. En términos argumentativos los autores de la TME sostienen que:

Metabolism has usually been incorporated into food web theory only to the extent of showing that the fluxes of energy and materials obey the laws of thermodynamics and conservation of energy, mass, and stoichiometry. It should be possible to do much more, in particular to use metabolic theory to understand the abundance, biomass, energy use, and elemental chemical composition of species populations or entire functional groups in terms of the effects of body size, temperature, and stoichiometry on metabolic rate. (Brown et al. 2004a, p.1784)

Brown y colaboradores, presentan aquí a la TME como una vía posible para ofrecer explicaciones de ciertos aspectos centrales de los ecosistemas, tales como la biomasa o el uso de energía entre los organismos y el ambiente. Entre los ejemplos que se ofrecen se puede mencionar a la estimación del "stock de biomasa en pie" [W]. Normalmente la biomasa en pie, puede estimarse como el peso por unidad de área [W $=\mathrm{g} / \mathrm{m}^{2}$ ] [ec. 8] (Odum 1994; Odum y Barrett 2006). Ahora bien, en el contexto de la TME, dicha estimación podría realizarse nuevamente por un modelo empírico análogo a los utilizados en la ecología de poblaciones y la ecología de comunidades, esto es, recurriendo a la ecuación de la tasa metabólica [ec. 3]. En este sentido, se menciona:

Principia 21(1): 99-123 (2017). 
It is straightforward to derive an expression for standing stock biomass. Eq. $\left[K \sim[R] M^{-3 / 4} e^{E / k T}\right]$ gives the effects of body mass and temperature on equilibrium population density (number of individuals per unit area). Multiplying this expression by the body size per individual, $M$, gives the corresponding equation for standing stock or stored biomass, $W$, per unit area: $\left[W \sim[R] M^{1 / 4} e^{E / k T}\right]$. The rate of supply of limiting resource, $[R]$, has direct linear effects on both carrying capacity and biomass. Total biomass increases nonlinearly with increasing body size and decreasing temperature. Large and/or cold organisms retain more resources in their bodies because they flux them more slowly through their metabolic pathways, and vice versa for small and/or hot organisms. (Brown et al. 2004a, pp.1782-3)

De esta manera, la estimación del valor de biomasa en pie [ec. 8] es reemplazado por un modelo alternativo [ec. 9] que describe y explica como varia la biomasa en relación con los dos factores antes presentados, la masa y la temperatura corporal:

$$
\text { [ec. 8] }\left[W=g / m^{2}\right] \triangleright\left[W \sim[R] M^{1 / 4} e^{E / k T}\right][\text { ec. 9] }
$$

Alcanzado este punto, estamos en condiciones de reconocer que los factores y reglas usados para dar cuenta de los diferentes dominios indagados - sea el dominio poblacional, el comunitario o el ecosistémico - son los mismos que fueron utilizados para caracterizar el metabolismo del organismo. Así, mediante ciertas modificaciones que sufre la ecuación de la tasa metabólica $\left[I=i_{0} M^{3 / 4} e^{-E / k T}\right.$ ] [ec. 3], inherente al dominio fisiológico, se construyen diferentes modelos dirigidos a los distintos dominios de la ecología (véase Tabla 1). Vale agregar que todos estos modelos "metabólicos", tienen en común la masa, la temperatura del organismo y también, las reglas que estos factores guardan con el metabolismo.

\begin{tabular}{ccc}
\hline Ecuaciones Dominios & Ecuaciones “clásicas" & Ecuaciones "metabólicas" \\
\hline Fisiológico & $Y=Y_{0} M^{b}$ [ec. 1] & $I=I_{0} M^{3 / 4} e^{-E / k T}$ [ec. 3] \\
& $e^{-E / k T}$ [ec. 2] & \\
Poblacional & $d N / d t=0$ [ec. 5] & $K \sim[R] M^{-3 / 4} e^{E / k T}$ [ec. 6] \\
Comunitario & - & $B \sim M^{-1 / 4} e^{-E / k T}$ [ec. 7] \\
Ecosistémico & $W=g / m^{2}$ [ec. 8] & $W \sim[R] M^{1 / 4} e^{E / k T}$ [ec. 9] \\
\hline
\end{tabular}

Tabla 1. Se presentan las ecuaciones "clásicas" y aquellas generadas desde la TME para cada dominio indagado. Obsérvese que las ecuaciones 6, 7 y 9 son análogas a la ecuación 3.

Principia 21(1): 99-123 (2017). 


\title{
5. Argumentos a favor de la reducción sustantiva de Sarkar: jerarquía abstracta
}

Habiendo precisado los argumentos que conciernen al criterio de fundamentalismo, en esta sección nos dirigiremos al criterio de jerarquía abstracta. En el primero apartado desarrollaremos la idea de jerarquía ecológica o funcional construida con un criterio que es considerado legítimo dentro de la bibliografía especializada (sección 5, apartado 1). Posteriormente, en el segundo apartado, destacaremos que el sentido de la explicación va de los niveles inferiores a los niveles superiores de la jerarquía considerada (5.2).

\subsection{Jerarquía ecológica construida con un criterio independiente}

Precisados los factores y las reglas del dominio fisiológico, que buscan dar cuenta de otros dominios no fundamentales (los ecológicos), resulta conveniente ahora volver a las sugerencias de Sarkar y organizar dichos dominios en una jerarquía abstracta. Con esta intención, dos jerarquías pueden ser consideradas centrales al seno de la biología: la jerarquía genealógica y la jerarquía ecológica o funcional (Morrone 2004; Folguera 2011). Es sobre ésta última que se organizan los dominios aquí estudiados y sus respectivos niveles: el dominio de la fisiología y su nivel individual, la ecología de poblaciones y su nivel poblacional, el dominio de la ecología de comunidades y su nivel comunitario y finalmente, la ecología de ecosistemas y su nivel ecosistémico. La construcción de la jerarquía ecológica, como sugiere Sarkar, debe asumir la elección de un criterio que dé cohesión a los niveles considerados. En relación con ello, Niles Eldredge (1985) sostiene que:

\begin{abstract}
If we leave reproduction (information transfer) to the genealogical hierarchy as a matter of definition, it is precisely such energy-matter interaction that we are left with to define - actually, to impart cohesion to - ecological entities $[\ldots]$ where the basic elements of the ecological hierarchy are seen as molecules, organisms, populations, local ecosystems, and, finally, regional biotas. Accepting, however, the distinction between purely biotic units versus those that commingle the biotic and abiotic (e.g., ecosystems), it would perhaps be as well to revert to the following list of nested entities that form the ecological hierarchy: molecules, organisms, populations, communities, and regional biotas. But such a system raises the need to consider the abiotic world separately [...] For considering the abiotic world separately necessitates recognition of still another hierarchy. (p.167)
\end{abstract}

De esta larga cita, dos aspectos caben ser destacados a nuestros fines. El primero es que la cohesión de la jerarquía ecológica está dada por la transferencia de materia y energía que ocurre a través de los niveles de la jerarquía (individuo, población,

Principia 21(1): 99-123 (2017). 
comunidad y ecosistema). A partir de dicho criterio es posible indicar entonces, este aspecto común a todos los niveles de la jerarquía (Morrone 2004). El segundo aspecto a destacar de las palabras de Eldredge es que se pone en evidencia la dificultad que supone el nivel ecosistémico. Este nivel difiere del resto de los niveles involucrados, al contemplar tanto el componente biótico como el abiótico, mientras que el resto de los niveles sólo supone el componente biótico. De aquí que Eldredge sugiera sacar el nivel ecosistémico, quedando entonces: organismos, poblaciones, comunidades y biotas regionales. Esta dificultad que viene dada por la "naturaleza" del nivel ecosistémico, también obliga a reconocer que en todos los niveles se podría agregar el componente abiótico. Es decir, todos los niveles de la jerarquía guardan algún tipo de interacción con componentes abióticos. Por tal motivo, algunos autores sugieren una jerarquía de biosistemas que incluya ambos componentes (el biótico y el abiótico) (Odum 1994; Odum y Barrett 2006). Como resultado de esta jerarquía de biosistemas se elimina el nivel comunitario, quedando entonces sistemas de órganos, sistemas de organismos, sistemas de poblaciones y ecosistemas (ver Esquema 1).

Un planteo similar al de Odum y al de Eldredge puede reconocerse en Brown, uno de los mayores exponentes de la TME. Según este último, la jerarquía tradicional en ecología incluyó en general los niveles de organismos, poblaciones, comunidades, ecosistemas y biosfera. Con todo, Brown (2003) destaca no sólo el inconveniente indicado sobre la "naturaleza" del ecosistema sino también el hecho de que el nivel comunitario y el ecosistémico no tiene límites espacio-temporales precisos: “...comunidades y ecosistemas, son muy difíciles de caracterizar de manera consistente. Son definidos en términos de límites arbitrarios espaciales y temporales." (2003, p.52). Asimismo, agrega que ni la comunidad ni el ecosistema comparten información genética, ni se reproducen: "Finalmente, y lo más importante, las comunidades y los ecosistemas no tienen una integridad inherente o cohesividad como entidades biológicas". (Brown 2003, p.52). Aquí Brown no explicita si se trata de un traslado de los criterios de la jerarquía genealógica a la jerarquía ecológica. O bien, si se trata de una extrapolación de las características del nivel individual (o incluso del nivel celular) hacia los niveles supra-individuales, "transformando" a la jerarquía ecológica en una jerarquía de "individuos". Recordemos que es el individuo (y también las células) los que portan las características de cohesividad, reproducibilidad y límites espacio-temporales precisos (ver por ejemplo Ghiselin 1974; Hull 1978; Vrba y Gould 1986; Ereshefsky 1988; Lieberman y Vrba 1995; Sober 1996; Gould y Lloyd 1999; Gould 2004). Esta última opción, referida a la extrapolación de las características del nivel individual, parece más acorde con la estrategia generada desde la TME, la cual toma al dominio fisiológico por fundamental. Más allá de esta perplejidad, Brown sugiere modificar la jerarquía ecológica tradicional, cambiando el nivel comunitario por el nivel especifico y el nivel ecosistémico por linajes monofiléticos, quedando entonces la siguiente jerarquía: organismos, poblaciones, especies, linajes 
monofiléticos y biosfera. Como puede examinarse, no hay total acuerdo en la bibliografía especializada acerca de los niveles involucrados en las jerarquías ecológicas (en este sentido, también se pueden reconocer diferentes jerarquías por ejemplo, en Valentine 1973 y en MacMahon et al. 1978).

En síntesis, sugerimos que aunque se reconozcan una serie de niveles usualmente involucrados cuando se piensa en términos jerárquicos (tales como el organismo, la población, la comunidad y el ecosistema) parece haber una falta de consenso para establecer una jerarquía única. Volveremos a esta controversia sobre las conclusiones.

\subsection{El sentido de la explicación en la jerarquía ecológica}

Habiendo presentado la idea de jerarquía ecológica o funcional, resulta preciso analizar el sentido de las explicaciones dentro de la misma. Siguiendo a Sarkar, se indaga entonces a partir de qué nivel/es se dan las explicaciones y hacia cuál/es se las dirige o si acaso se pueden reconocer explicaciones inherentes a cada nivel. Éste es sin duda un tema difícil de abordar dada la diversidad de dominios que aquí se están considerando. Con todo, se puede reconocer cierta tendencia en el sentido en que se han dado las explicaciones en ecología, donde se han privilegiado explicaciones que van desde los niveles inferiores hacia los niveles superiores de la jerarquía:

Cada nivel de integración implica una serie diferente y característica de atributos y problemas. Por ejemplo, una población tiene una densidad [... ] una propiedad que no se puede atribuir a un organismo individual. Una comunidad tiene una diversidad de especies, atributo que no tiene significado al nivel de población. En general, un científico con interés en un nivel particular de integración busca sus mecanismos de explicación en los niveles más bajos de integración y su significado biológico en los niveles más altos. Así, pues, para entender los mecanismos de cambio de una población, un ecólogo estudiará los mecanismos que operan sobre los organismos individuales y tratará de ver el significado de estos acontecimientos de la población en un marco de estudio de comunidades y ecosistemas. (Krebs 1986, p.26)

Siguiendo la descripción de Krebs, las explicaciones ofrecidas al seno de la jerarquía ecológica tienen un sentido preferencial que va de los niveles inferiores a los superiores. A continuación se representa esquemáticamente el sentido $(\rightarrow)$ en que usualmente se dan las explicaciones entre los niveles de la jerarquía:

$$
\text { [Órganos }] \rightarrow[\text { Organismo }] \rightarrow[\text { Población }] \rightarrow[\text { Comunidad }] \rightarrow[\text { Ecosistema }]
$$

A partir de aquí estamos en condiciones de sostener un tipo preferencial en el sentido de las explicaciones, que van "desde abajo hacia arriba”. Esta última afirmación es consistente con el sentido de las explicaciones generadas a partir de la TME. Recordemos que desde el dominio fisiológico y su nivel individual (ubicado en la sección 
inferior de la jerarquía) se generan las aproximaciones (mediadas por ecuaciones análogas a la ecuación metabólica [ec. 3]) a ciertas propiedades inherentes a los niveles poblacional, comunitario y ecosistémico. A la vez, estos niveles ecológicos, considerados en el contexto de una jerarquía, se encuentran siempre por encima del nivel individual.

\section{Argumentos a favor de la reducción sustantiva de Sarkar: jerarquía espacial}

La última de las exigencias sugerida por Sarkar para caracterizar la relación de reducción, se vincula con la idea de que a la jerarquía abstracta se le pueda aplicar el criterio físico de "ser una parte espacial de". Esto es, cuando una entidad del nivel inferior es una parte espacial menor, de una entidad de nivel superior.

\subsection{El criterio volumétrico en las entidades ecológicas}

El último criterio ofrecido por Sarkar, involucra examinar a las entidades en cuanto a su dimensión físicas, considerando más precisamente su espacialidad. En este sentido, debemos dejar de pensar en términos de niveles de la jerarquía para comprometernos con las entidades pertenecientes a cada nivel. Estas entidades físicas acorde con los dominios y los niveles ya mencionados serían: el organismo, la población, la comunidad y el ecosistema. El asunto puede ser delineado por medio del la siguiente interrogante ¿qué clase de relación espacial se presenta entre estas entidades ecológicas? Tal relación según Sarkar, no puede responder a cualquier clase, al menos si se quiere cumplir con su criterio de jerarquía espacial. Para este autor, debe presentarse una relación anidada esto es, que cada entidad sea una parte espacial menor de otra entidad mayor. Con otras palabras, se trata de convertir a la jerarquía abstracta en un sistema físico, donde las entidades se relacionan espacialmente a la manera de "muñecas rusas". Dicha idea no resulta novedosa al seno de la ecología y su jerarquía funcional, Elger ya en 1970 sugería que el desarrollo de la ecología moderna debería tomar dos direcciones: hacia el nivel infra-orgánico por un lado y por otro, hacia el nivel supra-orgánico (donde involucra por ejemplo los niveles poblacionales, comunitarios y ecosistémicos) (Naveh et al. 2001, p.88). Independientemente de que en su jerarquía se involucren más niveles de los aquí considerados, resulta interesante destacar el énfasis puesto en que la misma es: “[... ] un 'modelo' que ayuda a entender la naturaleza. Cualquiera de los nueves totales no es sino una parte de un total más grande, y está compuesto de partes que son por sí mismas totales menores" (Citado en: Naveh et al. 2001, p.88). En la cita de Elger, si bien se puede reconocer la relación de anidamiento, se debe destacar que aún se trata de un modelo más que

Principia 21(1): 99-123 (2017). 
de un sistema físico. Sin embargo, desde hace tiempo se ha pensado a la jerarquía ecológica en tanto sistema volumétrico. En esta linea argumental, Shultz que consideraba al ecosistema como el último nivel de la jerarquía, ${ }^{6}$ mencionaba a fines de 1960: "El objeto de estudio, en cualquier nivel, debe contener en el sentido volumétrico, los objetos del nivel inferior y debe por sí mismo ser una parte volumétrica de los niveles superiores [... ]" (Citado en: Naveh et al. 2001, p.93). De esta manera, el anidamiento espacial no es tratado únicamente como un modelo, sino que también fue considerado como un sistema volumétrico, o bien podríamos decir, un sistema físico. Así, a medida que ascendemos en la jerarquía, cada entidad incorpora nuevos organismos (de la misma especie cuando se pasa del nivel individual al poblacional o de especies diferentes cuando se pasa del nivel poblacional al comunitario) o bien, incorpora el componente abiótico (cuando se pasa del nivel comunitario al ecosistémico). De esta manera, la incorporación de estos ítems (sean nuevos organismos o el elemento abiótico) supone un agregado en el sentido físico, dando lugar a una relación espacialmente anidada.

Para lograr una mayor integración presentamos sucintamente en la Tabla 2, los principales resultados reconocidos para cada uno de los criterios sarkianos: fundamentalismo, jerarquía abstracta y jerarquía espacial.

\section{Conclusiones}

Llegando al final de nuestro recorrido estamos en condiciones de precisar el tipo de reducción alcanzado entre el dominio de la fisiología y los dominios de la ecología. Recordemos que Sarkar (1998) sugiere cinco tipos de reducción (resumidos en la tabla 3) en función del cumplimiento (+), no cumplimiento (-) o cumplimiento parcial (*) de los criterios aquí elaborados: fundamentalismo, jerarquía abstracta y jerarquía espacial. Los tipos de reducción sugeridos por este autor son: la "reducción débil" (o tipo 1), la "reducción jerárquica abstracta aproximada" (o tipo 2), la "reducción jerárquica abstracta" (o tipo 3), la "reducción fuerte aproximada" (o tipo 4) y la "reducción fuerte" (o tipo 5).

Como se puede notar en la Tabla 3, los tipos de reducción 2 y 4 se vinculan con el cumplimiento parcial del criterio de fundamentalismo. Ahora bien ¿qué significa en términos sarkianos, que el criterio de fundamentalismo se cumpla de un modo parcial? La respuesta a este interrogante se vincula, según Sarkar, con la idea de que la derivación que se hace desde el dominio fundamental (el fisiológico en nuestro caso) no se encuentra completamente justificada a partir de las reglas y factores considerados. Sarkar, cuestiona que este tipo de reducciones (tipos 2 y 4) puedan ser consideradas como "verdaderas explicaciones". Al volver sobre el recorrido trazado, podemos sugerir que los tipos 2 y 4 no parecen vincularse con lo arriba expuesto.

Principia 21(1): 99-123 (2017). 


\begin{tabular}{|l|}
\hline \multicolumn{1}{|c|}{ Fundamentalismo } \\
\hline Dominio explicativo \\
Fisiológico \\
Factores explicativos \\
Temperatura del organismo, masa corporal y metabolismo \\
Reglas explicativas \\
Relación metabolismo/temperatura y relación metabolismo/masa corporal \\
Dominios explicados \\
Ecología poblacional, ecología comunitaria y ecología ecosistémica \\
Propiedades explicadas \\
Tasa de crecimiento poblacional (en el equilibrio), patrones de diversidad \\
y estimación de biomasa \\
Justificación matemática \\
Modelo empírico que describe la relación del metabolismo individual con la \\
masa y con la temperatura corporal $\left[I=i_{0} M^{3 / 4} e^{-E / k T}\right]$ \\
\hline \multicolumn{1}{c}{ Jerarquía abstracta } \\
\hline Eriveles involucrados \\
Nivel individual, nivel poblacional, nivel comunitario y nivel ecosistémico* \\
priterio independiente \\
Transferencia de materia y energía entre componentes bióticos y entre \\
eomponentes bióticos y abióticos \\
Sentido de la explicación \\
De los niveles inferiores a los niveles superiores \\
\hline Entidades involucradas \\
\hline Individuo, población, comunidad y ecosistema \\
\hline
\end{tabular}

Tabla 2. Resumen de los principales resultados rastreados para cada uno de los criterios de reducción sustantiva: (a) fundamentalismo, (b) jerarquía abstracta y (c) jerarquía espacial. *Se debe recordar la falta de consenso sobre cuales niveles componen la jerarquía. **El anidamiento espacial queda supeditado a cuáles niveles componen la jerarquía abstracta. 


\begin{tabular}{|c|c|c|c|}
\hline $\begin{array}{c}\text { Criterios } \\
\text { Tipo de reducción }\end{array}$ & fundamentalismo & jerarquía abstracta & jerarquía espacial \\
\hline tipo 1 & + & - & - \\
tipo 2 & $*$ & + & - \\
tipo 3 & + & + & - \\
tipo 4 & $*$ & + & + \\
tipo 5 & + & + & + \\
\hline
\end{tabular}

Cinco tipos de reducción en función del cumplimiento (+), no cumplimiento ( - ) o cumplimiento parcial $(*)$ de los criterios analizados: fundamentalismo, jerarquía abstracta y jerarquía espacial.

En rigor, hemos podido precisar una serie de procedimientos dirigidos a desarrollar una justificación matemática para la derivación de diferentes ecuaciones aplicadas a los tres dominios ecológicos (el poblacional, el comunitario y el ecosistémico). Estas ecuaciones (la [6], [7] y [9] respectivamente) resultaron análogas a la ecuación del metabolismo [ec. 3]. En esta línea argumental, se sugiere que el criterio de fundamentalismo, se cumple de manera completa.

Para cumplir con el criterio de jerarquía abstracta, reconocemos una serie de inconvenientes que no pueden ser pasados por alto. Recordemos que parece haber consenso en el sentido de la explicación (que en nuestro caso se da desde los niveles inferiores hacia los superiores) (Krebs 1986). Además, también se reconoce cierto consenso en el criterio independiente que permite componer a los diferentes niveles en una jerarquía, recordemos que este criterio describe la transferencia de materia y energía entre los niveles (Eldredge 1985; Odum 1994; Odum y Barret 2006). Con todo, debemos destacar que este criterio supone no sólo la transferencia de materia y energía entre componentes bióticos sino también, entre componentes bióticos y abióticos. Esta aclaración es importante dado que pone en evidencia cierta inconsistencia. En los autores analizados - incluidos aquí algunos de los defensores de la TME parecen considerar únicamente una jerarquía de componentes bióticos, dejando de lado el componente abiótico. En rigor, el criterio de transferencia de materia y energía refiere a una jerarquía de biosistemas tal como fue presentada por Odum (1994), donde el criterio permite componer niveles que suponen ambos componentes, el biótico y el abiótico (ver Esquema 1). A este inconveniente acerca del criterio, se suma la falta de consenso en torno a los niveles involucrados en la jerarquía ecológica. En esta dirección señalamos que para Eldredge (1985) el ecosistema debe ser dejado fuera de la jerarquía por que incluye el componente abiótico. De igual manera Brown (2003), sugiere además que el nivel comunitario y el ecosistémico no tienen límites espacio-

Principia 21(1): 99-123 (2017). 
temporales precisos y que éstos no comparten información genética ni se reproducen. Siguiendo estas consideraciones, ni la comunidad ni el ecosistema pertenecerían a la jerarquía ecológica. Otra opción que se puede reconocer en la bibliografía es la de Odum (1994), quien propone una jerarquía de biosistemas (acorde con el criterio de transferencia de materia y energía) que incluye componentes bióticos y abióticos en todos los niveles, dando lugar a la exclusión del nivel comunitario. Así, al volver sobre el caso de reducción aquí indagado, tenemos un escenario complejo, ya que los niveles que componen la jerarquía abstracta no encuentran fácil solución entre los ecólogos.

En caso de aceptarse la jerarquía propuesta por Brown (2003), asumida implícitamente en la construcción de la TME (la cual recordemos involucra los niveles de organismo, población, especie, grupos monofiléticos y biosfera) estaríamos en los escenario de tipo 3 ó 5 (esto es una "reducción jerárquica abstracta" o una "reducción fuerte") según se cumpla (o no) el tercer criterio dado por Sarkar (ver Tabla 3). Pero como mencionamos, la jerarquía de Brown no cumple con el criterio independiente (por no contemplar el componente abiótico) y además, soslaya dos de los niveles (y dominios) más relevantes de la ecología, a saber: el nivel comunitario y el nivel ecosistémico. Estos niveles soslayados acarrean la perdida de cuerpos teóricos robustos que han estructurado el pensamiento ecológico hasta el presente, y en parte, la omisión de estos niveles explicaría la poca atención que ha recibido la jerarquía sugerida por Brown (Begon et al. 2006; Odum y Barret 2006). Ahora bien, ¿qué ocurre con la jerarquía usualmente aceptada en la ecología? Esta es: organismo, población, comunidad y ecosistema (representada prácticamente en todos los libros de texto). En este último caso persiste el problema de contar con un criterio que permita componer estos niveles de un modo coherente. Como fue mencionado, la incorporación del componente abiótico exigido por el criterio independiente que da cohesión a estos niveles, dejaría fuera de la jerarquía al nivel comunitario, dando lugar a la siguiente jerarquía: sistemas de organismos, sistemas de poblaciones y ecosistemas (ver Esquema 1). De esto se sigue que la ecología no parece presentar una jerarquía funcional consistente, que cumpla con la doble exigencia de tener un criterio independiente y a la vez, que dicho criterio no soslaye alguno (o algunos) de los niveles usualmente considerados por la disciplina. En resumen, resulta complejo establecer la jerarquía abstracta sugerida por Sarkar. De aquí que las reducciones de tipo 3 y 5 quedarían descartadas como descriptoras del caso aquí indagado.

Queda por pensar qué ocurre con el criterio de jerarquía espacial. En líneas generales parece que éste último criterio volumétrico puede ajustarse a la jerarquía abstracta. Es decir, se puede afirmar que las entidades involucradas (organismo, población, comunidad y ecosistema) cumplen con el criterio de ser una parte espacial menor de un total mayor. En esta línea argumental, no parece presentar inconvenientes este último criterio quedando sin embargo supeditado, a los niveles que efectiva- 
mente fueran incluidos en la jerarquía abstracta y también, quedando supeditado a si se trata de una jerarquía que incluye o no al componente abiótico.

Más allá de las posibilidades que ofrece la reducción en la versión de Sarkar, no se pueden obviar los argumentos desarrollados en la bibliografía especializada, en contra de la TME. No sólo se reconocen críticas a las predicciones derivadas de la TME y a las relaciones alométricas que dicha teoría considera sino también a la supuesta idea de que efectivamente se presente un esquema común en cuanto su funcionamiento energético (segunda sección). Esto es, a la idea de que efectivamente tengamos evidencias de que se presenta un metabolismo que resulte común o "universal" a todo lo vivo. Estas críticas no son menores, pues a pesar de que efectivamente se puedan construir modelos empíricos a partir de los factores y reglas sugeridos desde la TME, también es cierto que estos modelos distan de alcanzar algún grado de robustez (Véase: van der Meer 2006; Muller-Landau et al. 2006; White et al. 2007; Hawkins et al. 2007; Algar et al. 2007; O'Connor et al. 2007). Hechas estas consideraciones cabe preguntar: ¿estamos frente a un caso de reducción exitosa entre la fisiología y la ecología? Aquí no esta de más afirmar que la TME se encuentra fuertemente caracterizada por hipótesis del dominio fisiológico. Que la relación de reducción entre el dominio de la fisiología y los dominios de la ecología puede ser parcialmente formalizada por medio de la estrategia brindada por Sarkar y, que mediante los procedimientos presentados, se podría sugerir una relación de "reducción débil" (o tipo 1). Con todo, la falta de robustez en estos modelos y la crítica dirigida a la idea de un "metabolismo universal" sugieren cierta precaución a la hora de enfrentar una respuesta última. En rigor, este intento de reducción entre la ecología y la fisiología debe ser considerado en el contexto más amplio del ya desgastado programa reduccionista en biología. Cabe recordar aquí uno de los intentos no exitosos de reducción que ha recibido mayor atención en la filosofía de la biología, este es el caso de la genética clásica y su relación con la genética molecular. Es en este contexto más amplio que la propuesta de la TME no parece escapar de las variadas críticas que ya ha recibido el programa reduccionista en las ciencias de la vida.

\section{Referencias}

Algar, A. C.; Kerr, J. T.; Currie, D. J. 2007. A test of Metabolic Theory as the mechanism underlying broad-scale species-richness gradients. Global Ecology and Biogeography 16: 170-178.

Begon, M.; Townsend, C. R.; Harper, J. L. 2006. Ecology: from individuals to ecosystems. United Kingdom: Blackwell Publishing.

Brigandt, I.; Love, A. 2008. Reductionism in biology. In: Zalta, E. (ed.) The Stanford Encyclopedia of Philosophy. Stanford: Stanford University Press. Disponible en: http://plato.stanford.edu/entries/reduction-biology/. Acceso en: 24 abril 2017.

Principia 21(1): 99-123 (2017). 
Brown, J. H. 2003. Macroecología. México: Fondo de Cultura Económica.

Brown, J. H.; Gillooly, J. F; Allen, A. P.; West, G. B.; Savage, V. M. 2004a. Toward a metabolic theory of ecology. Ecology 85: 1771-1789.

- 2004b. Response to forum commentary on 'Toward a metabolic theory of ecology'. Ecology 85: 1818-1821.

Burian, R. 1996. Underappreciated pathways toward molecular genetics as illustrated by Jean Brachet's cytochemical embryology. In: S. Sarkar (ed.) The philosophy and history of molecular biology: new perspectives, pp.67-85. Dordrecht: Kluwer.

Carnap, R. 1934. The Unity of Science. London: Kegan Paul, Trench, Trubner, and Co.

Cottingham, K. L.; Zens, M. S. 2004. Metabolic rate opens a grand vista on ecology. Ecology 85: 1805-1807.

Darden, L.; Maull, N. 1977. Interfield theories. Philosophy of Science 44: 43-64.

di Pasquo, F. M.; Folguera, G. 2009. Tres dimensiones del reduccionismo en el contexto de la teoría metabólica ecológica. Principia 13: 51-65.

Dupré, J. 1993. The disorder of things: metaphysical foundations of the disunity of science. Cambridge: Harvard University Press.

Eldredge, N. 1985. Unfinished Synthesis. Biological Hierarchies and Modern Evolutionary Thought. Nueva York: Oxford University Press.

Ereshefsky, M. 1988. Individuality and macroevolutionary theory. Proceedings of the Biennial Meeting of the Philosophy of Science Association 1: 216-222.

Ferreira, M. J.; Folguera, G. 2015. Proliferación subdisciplinar en biología, debacle del reduccionismo y nuevas estrategias de unificación. Scientia Studiae 12(1): 121-135.

Folguera, G. 2008. La polisemia en la Biología: implicaciones científicas y epistemológicas. Revista Colombiana de Filosofía de la Ciencia IX(18 y 19): 155-164.

- 2011. Filosofía de la Biología. Análisis crítico de las jerarquías en la teoría de la evolución. España: Editorial Académica Española.

Folguera, G.; di Pasquo, F. M. 2011. Jerarquías ecológicas. Intentos reductivos de la fisiología a través de la macroecología. Ludus Vitalis XIX(36): 137-151.

Ghiselin, M. 1974. A radical solution to the species problem. Systematic Zoology 23: 536-544.

Gould, S. J. 2004. La estructura de la teoría de la evolución. Barcelona: Tusquets.

Gould, S. J.; Lloyd, E. A. 1999. Individuality and adaptation across levels of selection: how shall we name and generalize the unit of Darwinism? Proceedings of the National Academy of Sciences of the United States of America 96: 11904-11909.

Hawkins, B. A.; Albuquerque, F. S.; Araújo, M. B.; Beck, J.; Bini, L. M.; Cabrero-Sañudo, F. J.; Castro-Parga, I.; Diniz-Filho, J. A. F.; Ferrer-Castán, D.; Field, R.; Gómez, J. F.; Hortal, J.; Kerr, J. T.; Kitching, I. J. A.; León-Cortés, J. L.; Lobo, J. M.; Montoya, D.; Moreno, J. C.; Olalla-Tárraga, M.; Pausas, J. G.; Qian, H.; Rahbek, C.; Rodríguez, M. A.; Sanders, N. J.; Williams, P. 2007. A global evaluation of metabolic theory as an explanation for terrestrial species richness gradients. Ecology 88(8): 1877-1888.

Hull, D. L. 1972. Reduction in genetics-biology or philosophy? Philosophy of Science 39: 491499.

1978. A matter of individuality. Philosophy of Science 45: 335-360.

Kaiser, M. I. 2015. Reductive explanation in the biological sciences. Switzerland: Springer International Publishing.

Principia 21(1): 99-123 (2017). 
Kamp-Nielsen, L. 2008. Empirical Models. In: S. E. Jørgensen; B. D. Fath (eds.) Encyclopedia of ecology, pp.1239-1246. Amsterdam: Elsevier.

Kitcher, P. 1984. 1953 and all that: a tale of two sciences. The Philosophical Review 43: 335371.

Koehl, M. A. R.; Wolcott, D. B. 2004. Can function at the organismal level explain ecological patterns. Ecology 85(7): 1808-1810.

Kooijman, S. A. L. M. 2000. Dynamic Energy and Mass Budgets in Biological Systems. Cambridge: Cambridge University Press.

Krebs, C. J. 1986. Ecología. Análisis experimental de la distribución y abundancia. Madrid: Piramide.

Lieberman, B. S.; Vrba, E. S. 1995. Hierarchy theory, selection, and sorting. Bioscience 45: 394-399.

MacMahon, J. A.; Schimpf, D. J.; Andersen, J. C.; Shmith, K. G.; Bayn, Jr. R. L. 1978. Levels of biological organization: an organism-centere approach. Bioscience 28: 700-704.

Marquet, P. A.; Labra, F. A.; Maurer, B. A. 2004. Metabolic Ecology: Linking individuals to ecosystems. Ecology 85(7): 1794-1796.

Morrone, J. J. 2004. La zona de transición sudamericana: caracterización y relevancia evolutiva. Acta Ent. Chilena 28(1): 41-50.

Muller-Landau, H. C.; Condit, R. S.; Chave, J. Thomas, S. C.; Bohlman, S. A.; Bunyavejchewin, S.; Davies, S.; Foster, R.; Gunatilleke, S.; Gunatilleke, N.; Harms, K. E. Hart, T.; Hubbell, S. P.; Itoh, A.; Kassim, A. R.; LaFrankie, J. V.; Lee, S. H.; Losos, E.; Makana, J. R.; Ohkubo, T.; Sukumar, R.; Sun, I. F.; Supardi, N. M. N.; Tan, S.; Thompson, J.; Valencia, R.; Muñoz, G. V.; Wills, Ch.; Yamakura, T.; Chuyong, G.; Dattaraja, H. S.; Esufali, S.; Hall, P.; Hernandez, C.; Kenfack, D.; Kiratiprayoon, S.; Suresh, H. S.; Thomas, D.; Vallejo, M. I.; Ashton, P. 2006. Testing metabolic ecology theory for allometric scaling of tree size, growth and mortality in tropical forests. Ecology Letters 9: 575-588.

Nagel, E. 2006. La estructura de la ciencia. Barcelona: Ed. Paidós.

Naveh, Z.; Liberman, A. S. 2001. Ecología de Paisajes. Teoría y Aplicación. Buenos Aires: Facultad de Agronomía (UBA).

Nuñez, P. G.; Nuñez, M. A.; Ruggiero, A. 2007. Macroecología entre el reduccionismo y el dialogo. In: L. Salvatico; P. García (eds.) Epistemología e Historia de la Ciencia XIII, pp.404409. Argentina: Universidad Nacional de Córdoba.

O’Connor, M. P.; Kemp, S. J.; Agosta, S. J.; Hansen, F.; Sieg, A. E.; Wallace, B. P.; McNair, J. N.; Dunham, A. E. 2007. Reconsidering the mechanistic basis of the metabolic theory of ecology. Oikos 116: 1058-1072.

Odum, E. 1994. Ecología: El Vínculo Entre las Ciencias Naturales y las Sociales. México: Continental.

Odum, E.; Barrett, G. W. 2006. Fundamentos de Ecología. México: Cengage Learning.

Pigliucci, M.; Kaplan, J. 2006. Making sense of evolution: the conceptual foundations of evolutionary theory. Chicago: University of Chicago Press.

Rockwood, L. L. 2006. Introduction to Population Ecology. United Kingdom: Blackwell Publishing.

Rosenberg, A. 1985. The structure of biological science. Cambridge: Cambridge University Press.

Principia 21(1): 99-123 (2017). 
1994. Instrumental biology or the disunity of science. Chicago: University of Chicago Press.

Sarkar, S. 1998. Genetics and Reductionism. Cambridge: Cambridge University Press.

- 2001. Reduction: A Philosophical Analysis. Encyclopedia of Life Sciences. New York: John Wiley \& Sons.

- 2015. Nagel on reduction. Studies in History and Philosophy of Science 53: 43-56.

Sober, E. R. 1996. Filosofía de la biología. Madrid: Alianza Editorial.

Spicer J.; Gaston, K. 1999. Physiological diversity and its ecological implications. Oxford, United Kingdom: Blackwell Science.

Valentine, J. W. 1973. Evolutionary Paleoecology of the Marine Biosphere. Nueva York: PrenticeHall.

van der Meer, J. 2006. Metabolic theories in ecology. Trends in Ecology and Evolution 21(3): 136-140.

Vrba, E. S.; Gould, S. J. 1986. The hierarchical expansions of sorting and selection: sorting and selection cannot be equated. Paleobiology 12: 217-228.

White, R. C.; Cassey, P.; Blackburn, T. M. 2007. Allometric Exponents Do Not Support a Universal Metabolic Allometry. Ecology 88(2): 315-323.

Wimsatt, W. 1976. Reductive explanation: a functional account. In: R. Cohen (ed.) Boston studies in the philosophy of science n.30, pp.671-710. Dordrecht: Reidel.

Wimsatt, W. C.; Sarkar, S. 2006. Reductionism. In: S. Sarkar; J. Pfeifer (eds.) Philosophy of Science. An Encyclopedia, pp.696-703. Great Britain: Routledge.

FEDERICO DI PASQUO Facultad de Filosofía y Letras (FfyL) - CONICET - Investigador Asistente Grupo de Filosofía de la Biología Línea de investigación en epistemología y problemáticas ambientales dipasquof@ayhoo. com. ar

CHRISTIAN FRANCESE Doctorado en curso - FfyL Grupo de Filosofía de la Biología Línea de investigación en tecnociencia francese.christian@gmail.com

GUILlermo Folguera FfyL - CONICET - Investigador Adjunto Grupo de Filosofía de la Biología Director de Grupo guillefolguera@yahoo.com.ar

\section{Notas}

${ }^{1}$ Aquí es importante aclarar que Sarkar elude el problema de la explicación. Básicamente afirma que dada una explicación — no importan aquí las exigencias que esta supone — se

Principia 21(1): 99-123 (2017). 
debe focalizar en aquellos criterios adicionales que están dirigidos a reconocer una reducción: "The strategy adopted will be to assume that one is given an explanation, which qualifies as an explanation according to whatever explication that one may prefer, and then attempt to identify those additional criteria that the explanation must satisfy in order to be a reduction." (Sarkar 1998, p.9). Para una crítica de la postura de Sarkar en este sentido véase el trabajo realizado por Kaiser (2015).

${ }^{2}$ En las fuentes revisadas los autores de la TME parecen no distinguir entre tamaño corporal y masa. Para una critica sobre la polisemia del concepto de "tamaño corporal" véase Folguera (2008).

${ }^{3}$ El subrayado es nuestro.

${ }^{4}$ El subrayado es nuestro.

${ }^{5}$ Sobre los modelos empíricos en ecología: "An empirical model is based only on data and is used to predict rather than to explain systems. In ecology, such models often consist of mathematical functions like linear or nonlinear regressions which describe the trend of data." (Kamp-Nielsen 2008, p.1239).

${ }^{6}$ Vale destacar que en la jerarquía propuesta por Shultz se consideraban los componentes bióticos y también los abióticos.

\section{Apéndice}

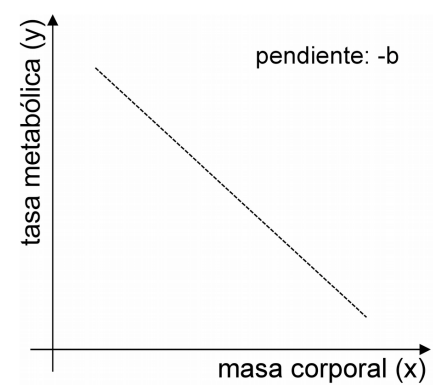

Figura 1. Representación esquemática de la relación (o la regla) dada entre la variable dependiente o explicada (eje $y$ ): la tasa metabólica y la variable independiente o explicativa (eje $x$ ): la masa corporal. A la vez, se destaca la pendiente negativa representada por $(-b)$. Como puede observarse un incremento en la masa se relaciona con una disminución en la tasa metabólica.

Principia 21(1): 99-123 (2017). 


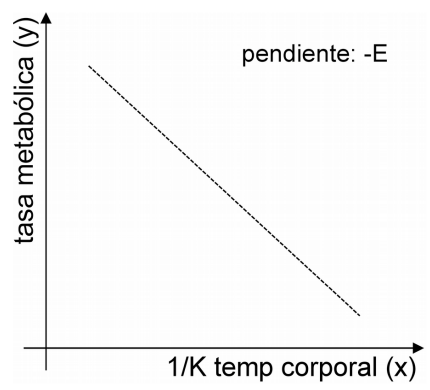

Figura 2. Representación esquemática de la relación (o la regla) dada entre la variable dependiente o explicada (eje $y$ ): la tasa metabólica y la variable independiente o explicativa (eje $x$ ): la inversa de la temperatura corporal. A la vez, se destaca la pendiente negativa representada por $(-E)$. Aquí, un incremento de la temperatura se relaciona con un aumento de la tasa metabólica.

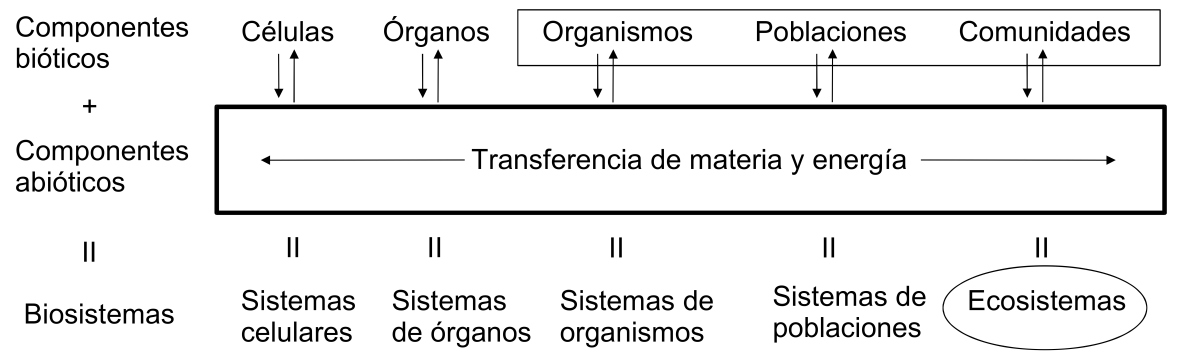

Esquema 1. Representación de los componentes bióticos, abióticos y del criterio que da cohesión a la jerarquía ecológica este es, la transferencia de materia y energía (esquema adaptado de Odum 1994 y de Odum y Barrett 2006).

Principia 21(1): 99-123 (2017). 